morbidity in adults however is not often reported in cases of empyema in paediatric populations. Associations include cystic fibrosis, malignancies, alcohol consumption and male gender.

Immunoglobulin levels and sweat test were normal for this patient, and there were no malignant cells in the microscopy report.

He was discharged in excellent condition. He remains under follow up with our team.

\section{A SYSTEMATIC REVIEW OF CLINICAL PREDICTION RULES TO DIAGNOSE BACTERIAL LOWER RESPIRATORY INFECTION IN CHILDREN IN PRIMARY CARE AND THEIR VALIDATION IN A NEW COHORT}

${ }^{1}$ Dermot Wildes*, ${ }^{2}$ Masters Chisale, ${ }^{1}$ Peter Harrington, ${ }^{3}$ Chris J Watson, ${ }^{1}$ Mark T Ledwidge ${ }^{1} J o e$ Gallagher. 'gHealth Research Group, UCD Conway Institute, School of Medicine, University College Dublin, Ireland; ${ }^{2}$ Mzuzu Central Hospital, Mzuzu, Malawi; ${ }^{3}$ WellcomeWolfson Institute for Experimental Medicine, Queen's University Belfast, UK

\subsection{6/archdischild-2021-europaediatrics.426}

Pneumonia is the greatest single cause of paediatric mortality. ${ }^{1}$ Children are perceived as a vulnerable population and it is well established that primary-care clinicians have a tendency to overprescribe antimicrobials, despite a low level of clinical suspicion for the presence of a bacterial respiratory infection ${ }^{2,3}$ Clinical prediction rules (CPR) combine variables derived from the history, examination and basic investigations to guide clinicians with a probability of a target diagnosis. ${ }^{4}$ Used correctly, CPRs can serve to reassure clinicians in their decision to avoid therapeutic intervention, adopting a 'watchand-wait' approach. ${ }^{5}$

This aim of this study was to identify existing clinical prediction rules for hospitalisation due to bacterial lower respiratory tract infection in children in primary care, with the aim to guide antibiotic therapy and to undertake validation of these rules in a novel cohort of children presenting to primary care in Malawi with World Health Organisation clinically defined pneumonia.

OVID MEDLINE \& EMBASE databases were searched for studies on the development, validation and clinical impact of clinical prediction models for bacterial LRTI in children between 1946 \& Q2-2021 Two reviewers screened all titles and abstracts independently. The study was conducted in accordance with the PRISMA guidelines. ${ }^{6}$

The BIOTOPE cohort (BIOmarkers TO diagnose PnEumonia) recruited children aged 2-59 months with WHO defined pneumonia from two primary care facilities in Mzuzu, Malawi. ${ }^{7}$ Validation of STARWAVe in BIOTOPE employed derivation and internal validation using bootstrapping.

1023 abstracts were identified and following the removal of duplicates, a review of 989 abstracts was conducted leading to the identification of one eligible model - the STARWAVE rule. $^{8}$ Validation of the STARWAVe rule was undertaken in the BIOTOPE cohort which consisted of 494 children with WHO clinically defined pneumonia presenting to primary care in Malawi. The area under the curve (AUC) of the STARWAVe rule for hospitalisation in BIOTOPE was found to be 0.8 (95\% C.I 0.75-0.85). However, the AUC of STARWAVe for a confirmed diagnosis of bacterial pneumonia was 0.39 (95\% C.I 0.25-0.54).

Clinical prediction rules could facilitate the advancement of antimicrobial stewardship in the area of paediatric pneumonia.
STARWAVe was accurate for predicting hospitalisation, but not bacterial infection. In the absence of a gold-standard indicator for bacterial LRTI, this is a reasonable surrogate and could lead to significant reductions in antibiotic prescription rates. Further work to determine its clinical impact is required.

\section{REFERENCES}

1. Katherine L O'Brien, Henry C Baggett, W Abdullah Brooks, Daniel R Feikin, Laura L Hammitt, Stephen RC Howie, Maria Deloria Knoll, Karen L Kotloff, Orin S Levine, Shabir A Madhi, David R Murdoch, J Anthony G Scott, Donald M Thea, Scott Zeger. Introduction to the epidemiologic considerations, analytic methods, and foundational results from the pneumonia etiology research for child health study. Clinical Infectious Diseases 15 June 2017;64(suppl_3):S179-S184. https://doi.org/ 10.1093/cid/cix142

2. Butler CC, Rollnick S, Pill R, Maggs-Rapport F, Stott N. Understanding the culture of prescribing: qualitative study of general practitioners' and patients' perceptions of antibiotics for sore throats. BMJ 1998;317:637-642

3. Cabral C, Lucas PJ, Ingram J, Hay AD, Horwood J. 'It's safer to ...'parent consulting and clinician antibiotic prescribing decisions for children with respiratory tract infections: an analysis across four qualitative studies. Soc Sci Med 2015;136$137: 156-164$

4. McGinn TG, Guyatt GH, Wyer PC, Naylor CD, Stiell IG, Richardson WS. Users' guides to the medical literature: XXII: how to use articles about clinical decision rules. Evidence-Based Medicine Working Group. Jama 2000 Jul 5;284(1):79-84

5. Fahey T, Van der Lei J. Producing and using clinical prediction rules.In: Knottnerus A, Buntinx $F$, eds. Evidence base of clinical diagnosis.Oxford: Blackwell Scientific 2008:213-36

6. Moher D, Liberati A, Tetzlaff J, Altman DG, Group P. Preferred reporting items for systematic reviews and meta-analyses: the PRISMA statement. PLoS Med 2009 Jul 21;6(7):e1000097.

7. Gallagher J, Chisale M, Das S, et al. Aetiology and severity of childhood pneumonia in primary care in Malawi: a cohort study. BMJ Open 2021:11:e046633. doi:10.1136/bmjopen-2020-046633

8. Hay $A D$, et al. 'Development and internal validation of a clinical rule to improve antibiotic use in children presenting to primary care with acute respiratory trac infection and cough: a prognostic cohort study.' The Lancet Respiratory Medicine $4(11): 902-910$

\section{IDIOPATHIC CHRONIC EOSINOPHILIC PNEUMONIA: A PAEDIATRIC CASE REPORT}

Srðan Banac*, Aleksandar Ovuka, Silvije Šegulja. Department of Paediatrics, Clinical Hospital Centre Rijeka, Croatia; Department of Paediatrics, Faculty of Medicine, University Rijeka, Croatia

\subsection{6/archdischild-2021-europaediatrics.427}

Introduction Rare pulmonary diseases in childhood continue to be a significant problem in clinical practice because there is a lack of diagnostic and therapeutic guidelines based on reliable scientific evidences. This group of diseases includes idiopathic eosinophilic pneumonias (IEP) that can manifest as acute or chronic or as transient Löffler syndrome. A paediatric case of chronic IEP was reported.

Case Report A 15-year old adolescent was referred to paediatric pulmonologist for suspected asthma. He complained of episodes of shortness of breath and wheezing for a year. Symptoms were more pronounced at night and were also effort induced. The symptoms showed a progressive trend The boy did not lose weight and had no fever nor night sweats. He denied smoking cigarettes.

Lung function was severely impaired (FVC 61\% FEV1 $47 \%$ ) with negative reversibility test. Inflammatory reactants were slightly elevated (ESR 28; CRP 11.1). Severe eosinophilia (3.36 x109/L) of peripheral blood was recorded. Chest CT demonstrated extensive bilateral ground-glass opacifications and peripheral airspace consolidations. Bronchoscopy revealed diffuse redness of tracheobronchial mucosa with severe BAL eosinophilia (eos 75\%). In spite to high total blood IgE level of $1265 \mathrm{kIU} / \mathrm{L}$, results of allergic and extensive microbiological 